\title{
Supporting Odour Source Localisation of Multi-Sniffer Robots with up-Wind Formation Repeater Network Topology
}

\author{
Kok Seng Eu, Kian Meng Yap, and Tiam Hee Tee
}

\begin{abstract}
Researchers have been working on odour tracking and sniffing robotics research, the purpose of the research is to replace sniffer dogs in Odour Source Localisation (OSL) task. Sniffer dogs have constraints in performing OSL task i.e. indentify the location of suspicious items such as explosives, illegal drugs or poison gas, it is actually risking the sniffer dogs' life. Thus far, odour tracking robotics research has shifted from single sniffer robot to multi-sniffer robots. It is because multi-sniffer robots can perform OSL task cooperatively over wireless network communication. Most of the multi-sniffer robots use star network topology which has a limitation of short exploration distance. It is constrained by the transmission distance range of a server transmitter i.e. a server transmitter with star network topology has a transmission distance limit; its clients (multi-sniffer robots) can only search within the transmission distance limit. This paper proposes a network topology that extends the exploration distance of multi-robots especially for multi-sniffer robots application. The proposed method has successfully extended the exploration distance by multiplying the transmission distance range with the total number of multi-sniffer robots.
\end{abstract}

Index Terms-Multiple robot systems, sniffer robots, odour source localisation, wireless network topology.

\section{INTRODUCTION}

In recent years, "Electronic Nose" technology has been an important development that can detect and recognize odours and smells. It is not only able to detect drugs and explosives but also detects cancer cell through the breath analysis of patient's lung [1]. Conversely, the biggest challenge in sniffer robot research is the sniffing strategy or sniffing algorithm. It is required to resolve the non-linearity of airflow in outdoor environment which could have turbulent airflow as it will cause a sniffer robot to derail or fail in its tracking of odour plume. Odour plumes are always stretched and twisted by the non-linearity turbulence airflow and causing odour plumes unpredictable and chaotic [2]. Therefore, OSL tasks are very difficult to be accomplished by using a single sniffer robot. To overcome the non-linearity problem of OSL task, researchers have proposed multi-sniffer robots' solutions [3]-[6]. The solutions can employ certain sophisticate sniffing algorithms

Manuscript received July 8, 2014; revised December 12, 2014. This work was supported by the Exploratory Research Grant Scheme (Grant No. ERGS /1/2012 /TK06 /SYUC /02/3) by Ministry of Higher Education in Malaysia and Sunway Internal Grant scheme (Grant No: INT-FST-CSNS-2013-13) at Sunway University, Malaysia.

Kok Seng Eu, Kian Meng Yap, and Tiam Hee Tee are with the Faculty of Science and Technology, Sunway University, Malaysia (e-mail: $\{12058889$, kmyap, 12056644\}@imail.sunway.edu.my). and integrated with multi-robot cooperative architecture. It enables multi-sniffer robots to communicate with each other through wireless network and accomplish OSL tasks cooperatively.

However, there is a need to find out the best wireless network topology for multi-sniffer robots, in order to maximize the chances of accomplishment of OSL tasks i.e. expand the search distance. Unlike other multi-robot systems, multi-sniffer robots have a certain characteristic during performing OSL tasks which is the formation of robots' coordination must be along to the up-wind direction of odour plume. This characteristic needs to be taken into consideration for designing the best network topology for multi-sniffer robots. Wrong design of network topology could cause short exploration distance of multi-sniffer robots and ineffective in performing OSL tasks.

In this paper, a network topology is proposed for multi-sniffer robots application in performing OSL tasks. The proposed topology is to extend the exploration distance of multi-sniffer robots. The remainder of this paper is organized as follows: Section II reviews the related work; Section III discusses the proposed method; Sections IV discusses the results and finally the conclusion of the works is presented in Section V.

\section{LITERATURE REVIEW}

The most common type of network topology used in multi-robot systems is star network topology [7]-[9]. It is due to its simple design and less development works are required. In star network topology, a main server communicates with all its clients (multi-robots). The communication pattern of star network is straight forward, where the main server is only communicating to a client at any one time, until the communication with that client is over, then only switch to next client. In brief, the communication is only between the main sever with its clients; clients are not able to communicate to each other in star topology.

The use of star network topology in multi-sniffer robots to perform OSL tasks has a limitation of short exploration distance. It is constrained by the transmission distance limit of a server transmitter; therefore, its clients (multi-sniffer robots) can only search within the transmission distance limit. If one of the sniffer robots goes beyond the transmission distance limit, the wireless communication could have poor performance.

Other than star network topology, mesh network topology is also a popular alternative for multi-robot systems. Many researchers have adopted mesh network topology for their 
multi-robot systems [10]-[12]. Mesh network not only allows a server communicate with its clients but also allows clients communicate to each other. Besides, it is able to allow dynamic communication sequences. However, the use of mesh network topology in multi-sniffer robots is overdesigned. It is because multi-sniffer robots have a certain characteristic during performing OSL tasks which is the formation of each sniffer robots that must be along to the up-wind direction of odour plume and most importantly the formation sequence of multi-sniffer robots is always static. In this case, a sniffer robot ' $\mathrm{A}$ ' is assigned at the position of most outlet of the searching distance, it is only able to communicate to sniffer robot " $\mathrm{B}$ " at all time; the sniffer robot " $\mathrm{B}$ " is assigned at the behind of sniffer robot " $A$ " to support it to search in longer distance. Therefore, the formation sequence is static and this characteristic makes the mesh network topology to be overdesigned, especially dynamic communication sequence feature that is very costly and it is not needed in multi-sniffer robots application.

\section{PROPOSED METHOD}

This paper proposes an optimal solution for supporting a network topology of multi-sniffer robots application in performing OSL tasks. The proposed method is to extend the exploration distance of multi-sniffer robots. Fig. 1 shows a multi-sniffer robots system with star network topology, every client (sniffer robot) must perform OSL task within the main server's transmission distance range. If a client (sniffer robot) moves beyond of the server's transmission distance range, the wireless communication between the server and the client could fail as shown in Fig. 2.

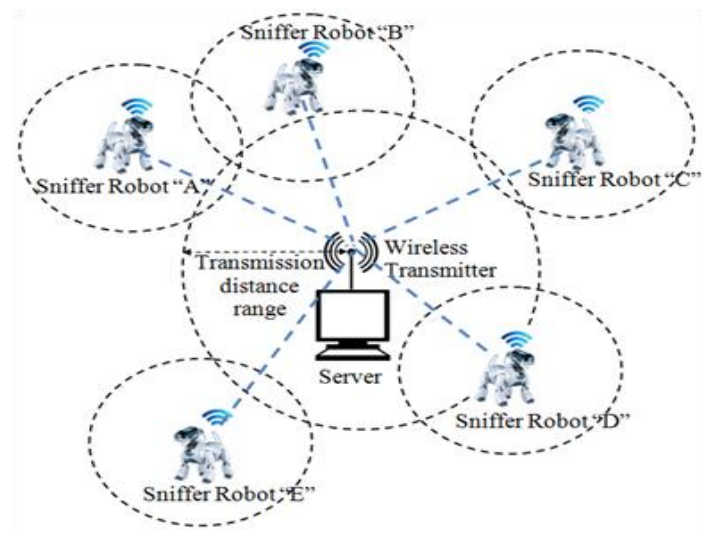

Fig. 1. Multi-robot system with star network topology.

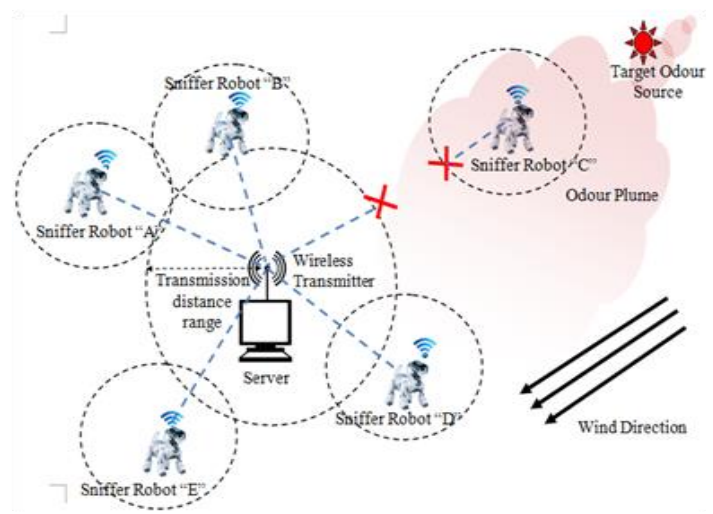

Fig. 2. Sniffer robot cannot go beyond the transmission distance range of server transmitter.
With star network topology, it has a wide exploration area but short exploration distance. It is a big disadvantage in OSL task application which does not require wide exploration area. In contrast, it requires narrow and long exploration distance. Multi-sniffer robots have a certain characteristic during performing OSL tasks which is the formation of each sniffer robots that must be along to the up-wind direction of odour plume, therefore narrow but longer exploration distance is needed.

The reason of this characteristic is caused by the odour plumes dispersal in the way that flow along wind direction which is narrow and long odour plume tails. It is described as Gaussian dispersion model as shown in Fig. 4 [13]. To identify the direction of long odour plume tail, it is required to detect the wind flow direction and it can be achieved by using ultrasonic anemometer which is able to accurately measure airflow below $0.01 \mathrm{~m} / \mathrm{s}$ [14]. If a multi-sniffer robot system with star network topology is employed, sniffer robot could not go along up-wind direction to track the odour plume because the rest of the sniffer robots in Fig. 2 are not supporting each other in OSL task to travel for longer exploration distance.

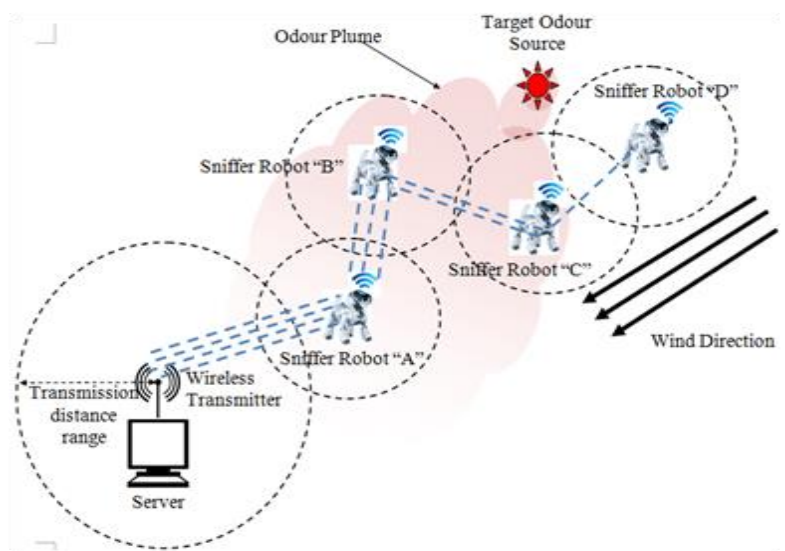

Fig. 3. Multi-sniffer robots with up-wind formation repeater network topology.

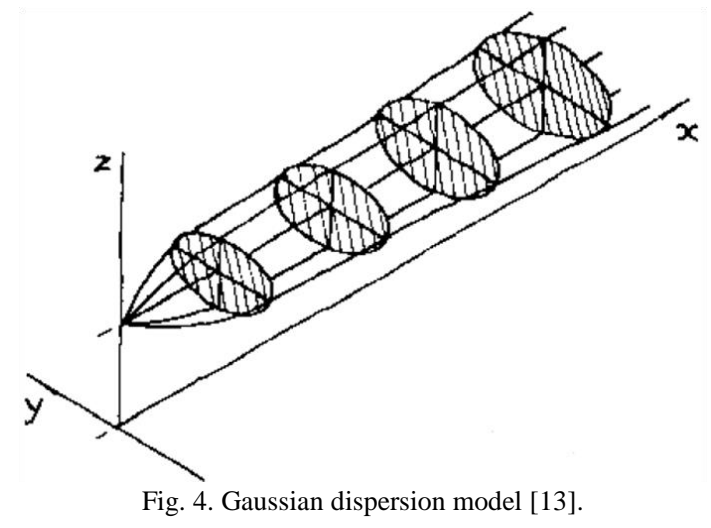

To overcome the problem, Up-Wind Formation Repeater network topology has been proposed. With this proposed network topology, each client (sniffer robot) can support each other to extend the distance of exploration. As illustrated in Fig. 3, the main server communicates with sniffer robot " $A$ ", and then sniffer robot "A" will communicate with " $\mathrm{B}$ ", sniffer robot " $\mathrm{B}$ " to " $\mathrm{C}$ " and so on so forth, until to the last client. Through the same way but reverse order, the last client is able to communicate back to the main server. It is important to 
highlight that the communication sequence of proposed network topology with multi-sniffer robots in Fig. 3, where it is showed in Fig. 5. First, the main server sends commands to sniffer robot " $A$ ", and then " $A$ " will echoing the commands to "B", "B" to "C", and "C" to "D". After that, sniffer robot "D" will reply its own status back to "C". "C" will reply back to "B" but reply two times for its own status and " $\mathrm{D}$ " status. So on and so forth, sniffer robot "A" will reply the main server for four times, which include all the sniffer robots status. By all means, the distance of exploration area has been extended because wireless communication distance has been increased.

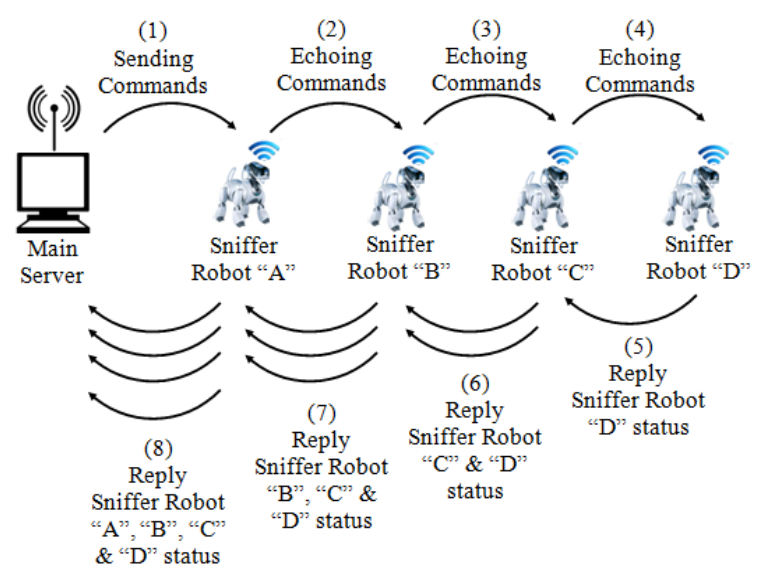

Fig. 5. The communication sequence of multi-sniffer robots with up-wind formation repeater network topology.

With the proposed method, the exploration distance of multi-sniffer robots can be extended as (1), where Total Exploration Distance (TED) is equal to the sum of Server Transmission Distance Range (TDRs) with the total summation of $\mathrm{N}$ number of Client Transmission Distance Range (TDRc).

$$
T E D=T D R_{s}+\sum_{n} T D R_{c}
$$

where $T E D=$ Total Exploration Distance, $T D R_{s}=$ Server Transmission Distance Range; $T D R_{c}=$ Client Transmission Distance Range; $N=$ Number of Client Robots.

\section{Discussion OF RESUlts}

The wireless communication devices employed in the experiments are Zigbee modules. Just like other wireless communication devices, the packet arrival rate of the Zigbee modules drops as the transmission distance increases. The first experiment is to find out the best transmission distance of the wireless communication device, which is the maximum transmission distance that still within an acceptable performance of packet arrival rate. As for rule of thumb, an acceptable performance of packet arrival rate has to be greater than $\pm 95 \%$ or packet loss has to be less than $\pm 5 \%$ [15]. Table I shows that when the transmission distance is greater than 5 meters, the packet arrival rate has dropped until unaccepted performance. Therefore, the transmission distance of 4 meters with the packet arrival rate of $98.80 \%$ has been chosen as the best transmission distance. This will be the interval distance between each client for the following experiments.
TABLE I: TRANSMISSION PERFoRMANCE-PACKET ARRIVAL RATE VERSE DISTANCE

\begin{tabular}{|c|c|}
\multicolumn{2}{c}{ DISTANCE } \\
$\begin{array}{c}\text { Transmission } \\
\text { Distance (meters) }\end{array}$ & $\begin{array}{c}\text { Packet Arrival } \\
\text { Rate }(\%)\end{array}$ \\
\hline 1 & 100 \\
\hline 2 & 100 \\
\hline 3 & 99.20 \\
\hline 4 & 98.80 \\
\hline 5 & 82.80 \\
\hline 6 & 76.20 \\
\hline 7 & 71.40 \\
\hline \multicolumn{2}{|c|}{}
\end{tabular}

The second experiment is to test the performance of star network topology for multi-sniffer robots. In the experiment, the total number of client is five and each client has an interval distance of 4 meters (according the best transmission distance of Table I), where client $1,2,3,4 \& 5$ are located at transmission distance of $4,8,12,16 \& 20$ meters. Table II shows the packet arrival rate of star network topology is dropped beyond unacceptable range after transmission distance of 8 meters, which it has only $81.40 \%$ packet arrival rate or packet loss of $18.60 \%$. The packet arrival rate is further dropping as the transmission distance increase.

TABLE II: STAR NETWORK TOPOLOGY PERFORMANCE

\begin{tabular}{|c|c|c|}
\hline $\begin{array}{c}\text { Total Number of } \\
\text { Client }\end{array}$ & $\begin{array}{c}\text { Transmission } \\
\text { Distance (meters) }\end{array}$ & $\begin{array}{c}\text { Packet Arrival } \\
\text { Rate }(\%)\end{array}$ \\
\hline 1 & 4 & 98.60 \\
\hline 2 & 8 & 81.40 \\
\hline 3 & 12 & 75.00 \\
\hline 4 & 16 & 65.00 \\
\hline 5 & 20 & 56.30 \\
\hline
\end{tabular}

The third experiment is to test the up-wind formation repeater network topology for multi-sniffer robots. In this experiment, there are five clients and each client is located in the same position as experiment 2 . Table III shows the packet arrival rate maintains within $\pm 95 \%$ even though the increase of the number of client and transmission distance. However, the packet arrival rate has slight dropped as the number of client increase. As compared to Table II, the packet arrival rate of Table III has made a significant improvement because the packet loss is within $\pm 5 \%$, which is within acceptable performance range.

TABLE III: THE PROPOSED UP-WIND FORMATION REPEATER NETWORK TOPOLOGY PERFORMANCE

\begin{tabular}{|c|c|c|}
\hline $\begin{array}{c}\text { Total Number } \\
\text { of Client }\end{array}$ & $\begin{array}{c}\text { Transmission } \\
\text { Distance (meters) }\end{array}$ & $\begin{array}{c}\text { Packet Arrival } \\
\text { Rate }(\%)\end{array}$ \\
\hline 1 & 4 & 98.60 \\
\hline 2 & 8 & 97.40 \\
\hline 3 & 12 & 96.10 \\
\hline 4 & 16 & 95.50 \\
\hline 5 & 20 & 95.10 \\
\hline
\end{tabular}

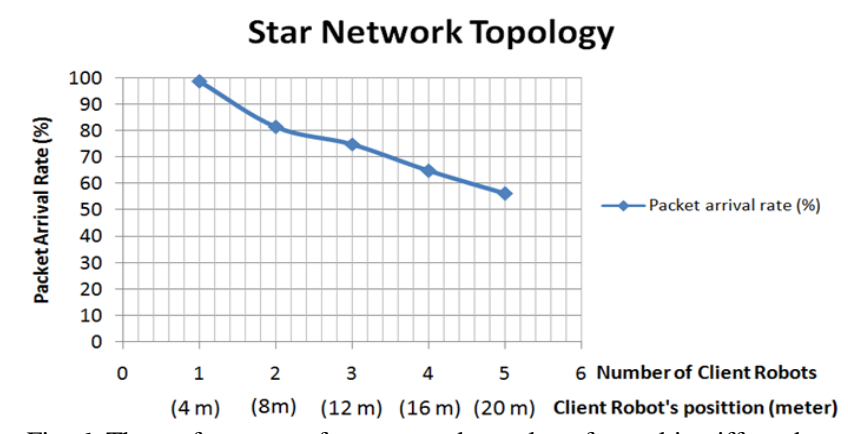

Fig. 6. The performance of star network topology for multi-sniffer robot. 


\section{Up-Wind Formation Repeater Network} Topology

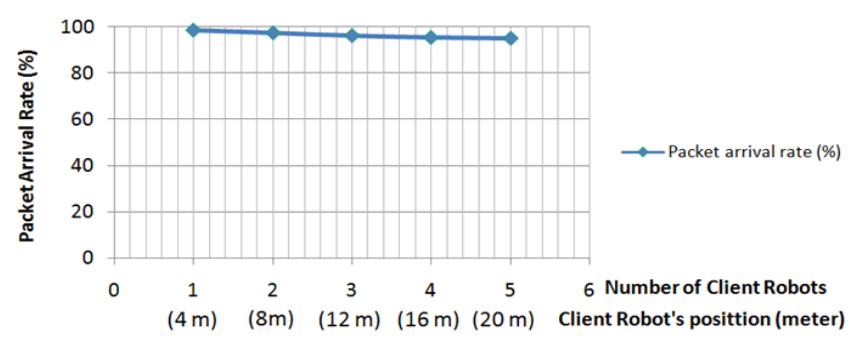

Fig. 7. The performance of up-wind formation repeater network topology for multi-sniffer robot.

Fig. 6 and Fig. 7 show the graph of number of the client $\&$ transmission distance versus packet arrival rate for star network topology and up-wind formation repeater network topology. Fig. 6 shows the significant improvement of packet arrival rate even with longer transmission distance.

\section{CONCLUSION}

Multi-sniffer robots have a certain characteristic during performing OSL tasks which is the formation of each sniffer robot that must be along to the up-wind direction of odour plume. This characteristic has been taken into consideration when designing the best network topology for multi-sniffer robots. Based on the conducted experiments, the proposed method has successfully extended the exploration distance of multi-sniffer robots by multiplying the transmission distance range with the total number of clients (multi-sniffer robots). Besides, it also maintains the packer arrival rate of the wireless communication. In future work, the proposed method can be implemented in movable repeating router networking system, whereby using multi-robots as movable repeating router to deliver wireless signals to some narrow and cavernous restricted environments such as cave, underground basement, tunnel and etc.

\section{ACKNOWLEDGMENT}

This work was supported by the Exploratory Research Grant Scheme (Grant No. ERGS /1/2012 /TK06 /SYUC /02/3) by Ministry of Higher Education in Malaysia and Sunway Internal Grant scheme (Grant No: INT-FST-CSNS-2013-13) at Sunway University, Malaysia.

\section{REFERENCES}

[1] V. Tran, H. P. Chan, M. Thurston, and P. Jackson, "Breath analysis of lung cancer patients using an electronic nose detection system," IEEE Sens. J., vol. 10, no. 9, pp. 1514-1518, 2010.

[2] H. Ishida, Y. Wada, and H. Matsukura, "Chemical sensing in robotic applications: A review,” IEEE Sens. J., vol. 12, no. 11, pp. 3163-3173, Nov. 2012.

[3] M. Turduev, G. Cabrita, M. Kırtay, V. Gazi, and L. Marques, "Experimental studies on chemical concentration map building by a multi-robot system using bio-inspired algorithms," Auton. Agent. Multi. Agent. Syst., vol. 28, no. 1, pp. 72-100, Dec. 2012.

[4] M. S. Couceiro, F. M. L. Martins, R. P. Rocha, and N. M. F. Ferreira, "Mechanism and convergence analysis of a multi-robot swarm approach based on natural selection," J. Intell. Robot. Syst., Feb. 2014

[5] Q. Lu, Q.-L. Han, and S. Liu, "A finite-time particle swarm optimization algorithm for odor source localization," Information Sciences, 2014.
[6] A. Marjovi and L. Marques, "Optimal swarm formation for odor plume finding," IEEE Trans. Cybern., no. 99, pp. 1-14, 2014.

[7] E. $\mathrm{Nu}$ and L. Basa, "Adaptive control for the synchronization of multiple robot manipulators with coupling time-delays," in Proc. the IEEE/RSJ International Conference on Intelligent Robots and Systems (IROS), 2010, no. 1, pp. 3308-3313.

[8] N. S. Babu and C. Engineering, "Implementation of communication among multiple robots," Int. J. Electron., vol. 2, no. 3, pp. 30-35, 2012 .

[9] A. R. Mosteo, L. Montano, and M. G. Lagoudakis, "Multi-robot routing under limited communication range," in Proc. 2008 IEEE International Conference on Robotics and Automation, 2008, pp. 1531-1536.

[10] H. A. Kadir and M. R. Arshad, "Decentralized cooperative communication framework for heterogeneous multi agent system," $J$. Commun., vol. 9, no. 2, pp. 163-170, 2014.

[11] A. Hart, N. Pezeshkian, and H. Nguyen, "Mesh networking optimized for robotic teleoperation," in Proc. the SPIE Conferences, 2012, pp. 25-27.

[12] C. Q. Nguyen, B.-C. Min, E. T. Matson, A. H. Smith, J. E. Dietz, and D. Kim, "Using mobile robots to establish mobile wireless mesh networks and increase network throughput," Int. J. Distrib. Sens. Networks, vol. 2012, pp. 1-13, 2012.

[13] J. Murtis, J. Murlis, J. S. Elkinton, and R. T. Carde, "Odor plumes and how insects use them," Annu. Rev. Entomol, vol. 37, no. 86, pp. 505-532, 1992.

[14] H. Ishida, T. Ushiku, S. Toyama, H. Taniguchi, and T. Moriizumi, "Mobile robot path planning using vision and olfaction to search for a gas source," IEEE Sensors, pp. 1112-1115, 2005.

[15] C. Padhye, K. J. Christensen, and S. Florida, "A new adaptive fec loss control algorithm for voice over ip applications," in Proc. IEEE International Performance, Computing and Communication Conference, 2000, pp. 307-313.

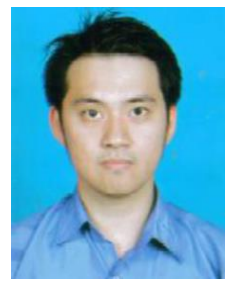

Kok Seng Eu is a robotics lecturer in Tunku Abdul Rahman University College and currently he is pursuing his $\mathrm{PhD}$ degree in computing from Sunway University, Malaysia. He had completed his master degree and BEng degree from Sheffield Hallam University. His interest areas of research are robotics, artificial intelligence systems, automation system and control.

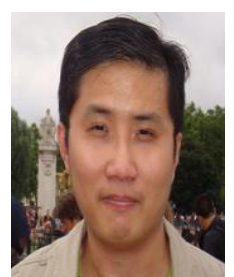

Kian Meng Yap received a diploma in electronic technology from Tunku Abdul Rahman University College (Malaysia) in 1997 and a master's degree with distinction from Queen's University Belfast in 2002. He passed the Engineering Council Examination parts 1 and 2 in 2000 and became a member of IET. He obtained his PhD degree also from Queen's University Belfast in 2008 on research into providing network quality of service (QoS) for haptic traffic over the distributed virtual environment (DVE) in a human-computer interface (HCI) environment. He is currently a member of IEEE. He has been employed at Sunway University in Malaysia since 2009 and is currently an associate professor at the Department of Computer Science and Networked Systems there. He has spent 10 years working in the machine control system, telecommunications, and computer networking industries. He is currently the principal investigator for three projects that are supported by the Ministry of Higher Education (MoHE) and the Sunway University Internal Grant. His current research interests include distributed haptics, HCI, network architectures and protocols, computer and telecommunications networks, haptics for visually impaired children, QoS architectures, virtual reality, embedded systems (microcontroller), and tele-robotics.

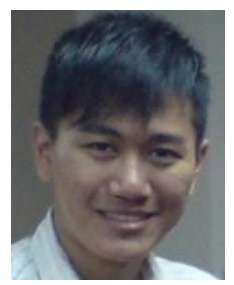

Tiam Hee Tee received the first class honour degree in computer and network technology from Northumbria University, UK in 2012. After receiving scholarship from Sunway University, he continues studying his master degree in computer science with major in tele-haptics and tele-robotics. He was a research assistant of Affective \& Assistive Technology Research Centre at the Sunway University. His research interests focus in the area of tele-operation, network communication, and human-robot-interaction. 